\title{
Zoledronate extends healthspan and survival via the mevalonate pathway in a FOXO-
}

\section{dependent manner}

Zhengqi Chen ${ }^{1}$, Julia Cordero ${ }^{2} \mathrm{PhD}$, Adel M Alqarni ${ }^{4}$, Cathy Slack ${ }^{3} \mathrm{PhD}$, Martin P Zeidler ${ }^{4} * \mathrm{PhD}$ and Ilaria Bellantuono ${ }^{1} * \mathrm{MD}, \mathrm{PhD}$

${ }^{1}$ Healthy Lifespan Institute, Department of Oncology and Metabolism, The Medical School, Beech Hill Road, University of Sheffield, Sheffield S10 2RX

${ }^{2}$ Institute of Cancer Sciences, University of Glasgow, Beatson Institute for Cancer, Research Switchback Rd, Bearsden, Glasgow G61 1QH

${ }^{3}$ School of Life \& Health Sciences, Aston University, Aston Triangle, Birmingham B4 7ET

${ }^{4}$ Department of Biomedical Science, The University of Sheffield, Western Bank, Sheffield S10 2TN

*These authors contributed equally

(C) The Author(s) 2021. Published by Oxford University Press on behalf of The Gerontological Society of America.

This is an Open Access article distributed under the terms of the Creative Commons Attribution License (http://creativecommons.org/licenses/by/4.0/), which permits unrestricted reuse, distribution, and reproduction in any medium, provided the original work is properly cited. 
Corresponding author

Ilaria Bellantuono

Professor in Musculoskeletal Ageing

Healthy Lifespan Institute

Department of Oncology and Metabolism

D Floor

The Medical School

Beech Hill Road

Sheffield S10 2RX

Telephone: +44 (0)1142159037

Fax: +44 (0)114271 2475

Email i.bellantuono@shef.ac.uk 


\section{ABSTRACT}

Over recent decades, increased longevity has not been paralleled by extended healthspan, resulting in more years spent with multiple diseases in older age. As such, interventions to improve healthspan are urgently required. Zoledronate is a nitrogen containing bisphosphonate, which inhibits the farnesyl pyrophosphate synthase (FPPS) enzyme, central to the mevalonate pathway. It is already used clinically to prevent fractures in osteoporotic patients, who have been reported to derive unexpected and unexplained survival benefits. Using Drosophila as a model we determined the effects of Zoledronate on lifespan, parameters of healthspan (climbing ability and intestinal dysplasia) and the ability to confer resistance to oxidative stress using a combination of genetically manipulated Drosophila strains and Western blotting. Our study shows that Zoledronate extended lifespan, improved climbing activity and reduced intestinal epithelial dysplasia and permeability with age. Mechanistic studies showed that Zoledronate conferred resistance to oxidative stress and reduced accumulation of X-ray-induced DNA damage via inhibition of FPPS. Moreover, Zoledronate was associated with inhibition of pAKT in the MTOR pathway downstream of the mevalonate pathway and required $\mathrm{dFOXO}$ for its action, both molecules associated with increased longevity. Taken together, our work indicates that Zoledronate, a drug already widely used to prevent osteoporosis and dosed only once a year, modulates important mechanisms of ageing. Its repurposing holds great promise as a treatment to improve healthspan.

Keywords aging, lifespan, bisphosphonates, DNA damage, Drosophila 


\section{INTRODUCTION}

It has been estimated that by 2050, Europe, North America, and eight countries across the other continents will have more than $30 \%$ of their population over the age of $60(1)$. However, this increase in life expectancy has not translated into extended healthspan(2). More than $60 \%$ of those over the age of 65 suffer from multimorbidities - defined as the co-existence of multiple chronic health conditions(3). These multimorbidities impose quality-of-life and care management challenges associated with treating multiple conditions individually and are frequently associated with increased costs, reduced efficacy and increased likelihood of adverse events due to polypharmacy(4). Targeting pathways underpinning central mechanisms of ageing, which are common to multiple disorders offers new opportunities to treat multimorbidity and overcome these problems.

Zoledronate (Zol) is a nitrogen containing-bisphosphonate used for the treatment of skeletal disorders, including osteoporosis. It inhibits the mevalonate pathway through inhibition of the enzyme farnesyl pyrophosphate synthase (FPPS)(5) and thereby inhibits bone resorption by inhibition of osteoclast activity. Due to its high affinity binding to hydroxyapatite crystal mineralisation, Zol is strongly enriched in bone and released following bone breakdown conferring long lasting activity(6). In clinical practice, Zol is administered by intravenous infusion once a year in post-menopausal women to prevent fractures $(7,8)$. Recently, retrospective analysis of several clinical trials showed that patients treated with Zol had a significant decrease in mortality rates(9). In addition, among patients admitted to intensive care units, those previously treated with Zol had increase survival despite overall higher level of multimorbidities and older age(10). It is unknown whether other mechanisms independent of bone protection may be involved in the Zol-dependent extension of survival.

The mevalonate pathway is an important metabolic pathway responsible for the production of cholesterol and protein prenylation. One key group of isoprenylated proteins are small GTPases(11), important signalling molecules which play central roles in multiple cellular processes, including 
cellular morphology, integrin function and longevity-associated pathways such as mTOR(12). In this study we examined whether Zol is able to extend lifespan and healthspan independent of its effect on bone using Drosophila, a model widely used for ageing studies. While retaining evolutionarily conserved components of both the mevalonate and mTOR pathways, Drosophila does not feature the bone-like mineralisation present in mammalian models(13). This approach allows us to exclude mechanisms, which have been hypothesized to explain Zol-mediated increases in human survival. These include the bone acting as a regulator of other tissues' homeostasis(10) through the release of hormones such as osteocalcin a regulator of glucose homeostasis(14). In doing so, our approach allows osteo-protective and gero-protective activities to be dissected in vivo. Our results uncovered a previously unrecognised role of Zol extending both lifespan and healthspan and improving survival in the presence of oxidative stress. The extension in survival by Zol is associated with a reduction in pAKT expression and requires dFOXO signalling, a conserved pathway well known for its positive effects on longevity.

\section{EXPERIMENTAL METHODS}

\section{Fly Stocks and Husbandry}

The white ${ }^{\text {Dahomey }}\left(w^{\text {Dah }}\right)$ stock has been maintained in large population cages with overlapping generations since $1970(15)$ and was a kind gift of the Partridge lab. $y^{1}, w^{67 c 23}$; $P\left(\right.$ lacW)Fpps $s^{k 06103} / C y O$ and $y^{1}, w^{67 c 23} ; \operatorname{raw}^{k 03514}, \quad P\left(\right.$ lacW)Fpps ${ }^{k 03514} / C y O$ (ref (16)) and $w^{1118} ;$ foxo ${ }^{494} / T M 6 B, T b^{1}(\operatorname{ref}(17))$ were obtained from the Bloomington Drosophila Stock Centre. w; Su(H)-lacZ, esg-Gal4,UAS-GFP/CyO expresses GFP in intestinal stem cells and progenitor cells and was a kind gift of Leanne Jones(18). $w^{+} ; G M R-G a l 4, U A S$-white ${ }^{R N A i}$ is a recombinant between GMR-Gal4 (ref(19)) and GD30033, an in vivo hairpin loop RNAi construct targeting white mRNA from the Vienna Drosophila Resource Center(20). 
All flies were maintained in a 12:12h light-dark cycle on standard yeast, molasses, cornmeal, agar food at $18^{\circ} \mathrm{C}$ or $25^{\circ} \mathrm{C}$. Drug-containing food was prepared using freshly cooked molten fly food cooled to $60^{\circ} \mathrm{C}$. Zol (kindly provided by Hal Hebetino, University of Rochester), GGOH (Sigma Aldrich, Dorset, U.K.) and FOH (Sigma Aldrich, Dorset, U.K.) or carrier(s) were added, mixed thoroughly and immediately poured into standard vials and bottles so as to minimize exposure of drug to high temperatures. The continued activity of Zol following brief exposure to this temperature was confirmed molecularly (data not shown). Drug food was stored at $4{ }^{\circ} \mathrm{C}$ until use for a maximum of 3 weeks.

For lifespan experiments overnight embryo collections from approximately 200 adult flies were collected on apple juice agar plates, washed with water and $32 \mu 1$ of embryos transferred into food bottles. Flies that subsequently ecclosed within the first $24 \mathrm{hrs}$ of the first to emerge were discarded with those subsequently ecclosing over an 16-20 hour overnight collection window being used for experiments. These later flies were transferred into new food bottles without the use of $\mathrm{CO}_{2}$ and incubated for 3-4 days. Flies were then sorted by gender and counted while minimizing $\mathrm{CO}_{2}$ exposure.

\section{Longevity Assay and $\mathrm{H}_{2} \mathrm{O}_{2}$ Survival Assays}

For longevity assays, 20 gender matched flies were maintained in vials of food and transferred into new vials every 2-3 days. At every transfer numbers of dead or censored flies were recorded. Unless specifically specified 100 flies were analysed for each experimental with three independent experimental replicates. To test survival in presence of hydrogen peroxide, adult flies of the appropriate genotype and pre-treatment were first starved in vials containing $1 \%$ agar for 3 hours and then transferred to standard food containing $5 \% \mathrm{H}_{2} \mathrm{O}_{2}$ and the appropriate drugs / vehicle. Death was then scored at 12, 24 and 36 hours and then every 
2 hours for the next 48 hours. If $100 \%$ mortality was not reached by that point, scoring was continued every 12 hours.

\section{Rapid iterative negative geotaxis (RING) assays}

For rapid iterative negative geotaxis (RING) assay flies we kept in groups of $\sim 150$. The day before an experiment, flies were sorted into groups of 20 and transferred into separate vials following brief $\mathrm{CO}_{2}$ anaesthesia. Flies were then transferred into $25 \mathrm{ml}$ strippette (Fisher Scientific) and after 1 minute, the strippette was tapped to knock flies to the bottom and initiate the negative geotaxic response. After 15 seconds a photograph was taken and the position of each fly, and hence the distance climbed, was recorded. Twenty flies were assessed from each condition with each experiment repeated 3 times. Flies that climbed above $10 \mathrm{~cm}$ were classified as 'high climbers'.

\section{Gut barrier integrity and food uptake assays}

For gut barrier integrity assays Drosophila food containing $2.5 \%(\mathrm{w} / \mathrm{v})$ erioglaucine disodium salt (Sigma Aldrich) and drugs (as appropriate) was fed to flies for 9 hours. For gut integrity assays flies were scored for uniform blue coloration beyond the GI tract ('Smurf-ness').

Food uptake assays were undertaken as described in (21) using food containing $1 \%(\mathrm{w} / \mathrm{v})$ erioglaucine disodium salt and $11.7 \mu \mathrm{M}$ Zol as appropriate. Ten adult flies between $8-11 \mathrm{~d}$ old were placed in vials for $22 \mathrm{~h}$ and allowed to feed $a d$ libitum at $25^{\circ} \mathrm{C}$. Flies were frozen and then dissociated in $\mathrm{dH}_{2} \mathrm{O}$ before centrifugation to pellet debris and extract colour from internalised food. In addition, colour present in faecal matter deposited on the walls of the vial was recovered by washing with $\mathrm{dH}_{2} \mathrm{O}$. Colour, and hence quantity of food consumed was calculated on the basis of OD $630 \mathrm{~nm}$ measurements and reference to a standard curve of dye dilutions (eFig 4A). Extracts from flies fed on undyed food were used as a blank. Ten groups 
of 10 flies were tested for each condition and gender and tested by one-way ANOVA. Final values are expressed as $\mu \mathrm{g}$ food per fly per day (correcting for the $22 \mathrm{~h}$ sampling period).

\section{DNA damage assay}

An overnight egg collection of $w^{+}$, GMR-Gal4 UAS-white ${ }^{R N A i}$ adults outcrossed to OreR were allowed to develop for 96 hours at $25^{\circ} \mathrm{C}$. These heterozygous third instar larvae were then irradiated with 200Gy using a Torrex Cabinet X-ray system (Faxitron X-ray, Arizona, USA) and adult flies that subsequently ecclosed were scored for the frequency of red $w^{+}$clones.

\section{Drosophila midgut analysis}

Midguts from $S u(H)$-lacZ; esg-Gal4,UAS-GFP/CyO female adults were dissected in cold PBS and fixed in PBS+4\% formaldehyde and blocked in PBST (PBS, 0.1\% Triton X-100 and 1\% BSA). Antibodies included Chicken anti-GFP 1:4000 (Abcam, Cambridge, UK) and antiPhospho-histone H3 1:100 (New England Biolabs, Ipswich, USA) and secondary antibodies AlexaFluor $^{\mathrm{TM}} 488$ goat anti-chicken and AlexaFluor $^{\mathrm{TM}} 594$ goat anti-rabbit IgG (Life Technologies, Oregon, USA) were used. Stained guts were mounted with Fluoroshield ${ }^{\mathrm{TM}}$ with DAPI (Sigma Aldrich) and Grace Bio-Labs SecureSeal ${ }^{\mathrm{TM}}$ imaging spacers. Samples were imaged using Perkin Elmer Spinning Disk confocal microscope with a 40X objective. Images were then processed using Image $\mathrm{J}$. 


\section{Protein extraction and western blot}

Thirty flies of the appropriate genotype were snap frozen in liquid $\mathrm{N}_{2}$ and crushed using an Eppendorf pestle in $200 \mu \mathrm{l}$ ice-old lysis buffer $(50 \mathrm{mM}$ Tris $\mathrm{HCl} \mathrm{pH} \mathrm{7.4,250mM} \mathrm{NaCl,} 5 \mathrm{mM}$ EDTA and $0.003 \%$ Triton X-100) with protease inhibitor (cOmplete Mini, EDTA-free, ROCHE, Sussex, UK). After 30mins incubation at $4^{\circ} \mathrm{C}$ extracts were centrifuged at $13,000 \mathrm{rpm}, 4^{\circ} \mathrm{C}$ for $30 \mathrm{mins}$ and supernatants stored at $-20^{\circ} \mathrm{C}$.

Proteins $(40-60 \mu \mathrm{g})$ were electrophoresed through $4-15 \%$ Mini-PROTEAN® TGX ${ }^{\mathrm{TM}}$ Precast Protein Gels (Bio-Rad, Hertfordshire, UK) and transferred onto polyvinylidene difluoride (PVDF) membrane (Immobilon-P Membrane, Millipore, Hertfordshire, UK) using standard techniques. Primary antibodies used were anti-pAKT 1:500, anti-AKT 1:500, anti-tubulin 1:1000 (Cell Signaling Technology, Denver, Massachusetts). HRP conjugated secondary antibodies were used at 1:10,000 and visualised using ECL (GE Healthcare, Buckinghamshire, UK), high performance chemiluminescence film (Amersham Hyperfilm ECL, GE Healthcare) and an Optimax 2010 X-ray film processor (PROTEC, Oberstenfeld, Germany).

\section{Statistical Analysis}

Statistical analysis was performed on GraphPad Prism 7. For single comparisons data were analysed using non-parametric unpaired Student's t-test. For multiple comparisons, ordinary one-way analysis of variance (ANOVA) was used followed by Sidak's multiple comparison post-hoc tests. For the analysis of RING assay Two Way ANOVA was used followed by Dunnet's multiple comparison test to determine the effect of dose of the treatment at the 
different ages. Survival data was analysed using Log-rank (Mantel-Cox) test to identify significant differences in organism survival between treatment groups. For the analysis of the Smurf assay Chi-squared test was used. All data is expressed as mean \pm standard deviation (SD). A difference was stated to be statistically significant if the $\mathrm{p}$ value was $<0.05\left({ }^{*} \mathrm{p}<0.05\right.$; $* * \mathrm{p}<0.01 ; * * * \mathrm{p}<0.001 ; * * * * \mathrm{p}<0.0001)$.

\section{RESULTS}

\section{Zol increases lifespan in Drosophila}

To determine whether Zol might have beneficial effects on the lifespan of Drosophila, both male and female $w^{D a h}$ flies were maintained on standard fly food supplemented with $1 \mu \mathrm{M}$ or $10 \mu \mathrm{M}$ Zol from day 4 of adult life onwards (Fig. 1 A\&B and eFig.1). In multiple experimental replicates, the survival of males fed with $1 \mu \mathrm{M}$ Zol was significantly increased compared to the vehicle-treated group (Fig. 1A and eFig.1). By contrast, females, showed no significant beneficial effect when treated with Zol at $1 \mu \mathrm{M}$ throughout their lives and $10 \mu \mathrm{M}$ of $\mathrm{Zol}$ had adverse effects on female survival (Fig. 1B).

As Zol modulates the mevalonate pathway through inhibition of FPPS, we next tested the lifespan of two heterozygous FPPS mutants $\left(F_{p p s} s^{k 06103} /+\right.$ and $\left.F_{p p s}{ }^{k 03514} /+\right)$ - two independently generated homozygous-lethal loss-of-function alleles containing transposon insertions within the FPPS gene ${ }^{15}$. Following at least 7 generations of outcrossing into the $w^{D a h}$ genetic background, both mutant strains showed a statistically significant increase in overall survival in males and females compared to $w^{D a h}$ controls (Fig. $1 \mathrm{C} \& \mathrm{D}$ and eFig2) suggesting that reduced FPPS activity mediates an extension in lifespan.

To limit potential side effects due to long term drug treatment, we next assessed the effects of Zol on lifespan when starting administration at day 40 of adult life (middle age) (red arrow in Fig. 1 E\&F and 
eFig.3). This late treatment led to a significant increase in survivorship in both males and females for both 1 and $10 \mu \mathrm{M}$ Zol compared to controls (Fig. $1 \mathrm{E} \& \mathrm{~F}$ and eFig.3). This lifespan extension was significant for both males and females with $1 \mu \mathrm{M}$ Zol producing average median lifespans increases of $1.79 \%( \pm 0.10 \%)$ and $11.53 \%( \pm 2.10 \%)(\mathrm{N}=3)$ for male and females respectively, while with $10 \mu \mathrm{M}$ Zol, survival was increased in males by $4.67 \%( \pm 1.03 \%)$ and females by $16.51 \%( \pm 4.94 \%)(\mathrm{N}=3)$. Finally, to verify that these results were not influenced by calorific restriction caused by unpalatable food we exposed the flies to drug-laced food containing a non-toxic food colorant via which the volume of food both present within flies and excreted can be measured. No significant difference in food uptake was observed in presence of Zol in either males or females (eFig. 4 n=10/trial, 10 trials per condition), excluding the possibility that the life extending effects were the result of caloric restriction.

\section{$\underline{\text { Zol increases healthspan in Drosophila }}$}

To determine whether Zol improved signs of healthspan we next tested its effects in Drosophila by assessing their ability to climb and by the presence of signs of intestinal epithelial dysplasia and intestinal permeability. Displaying strong negative geotactic responses, climbing activity is a widely used assay of Drosophila health and activity(22) and was assessed using the rapid iterative negative geotaxis (RING) assay (Fig. 2). We classed as "high climbers" flies able to climb over $10 \mathrm{~cm}$ in 15 seconds. As expected, the percentage of high climbers decreased significantly with age in both males (Fig. 2 A\&C) and females (Fig. 2 B\&D). However, a significant improvement in climbing ability was observed in both males and females receiving Zol from day 4 of adult life (Fig. 2 A\&B) or starting at 40 days of life (Fig. 2 C\&D) when as assessed at 42 and 56 days of age respectively. Two-way ANOVA analysis showed that, despite a significant interaction between age and treatment (Fig. 2 for details of statistical analysis), no significant effect of Zol was observed at a later time point in any of the conditions analysed by post hoc test. These data show there is an overall improvement in climbing ability at middle age, which is independent of the effects on lifespan. 
As flies age, intestinal barrier dysfunction and epithelial dysplasia develop in female flies, a development associated with increased mortality and a development considered to be a good marker of healthspan(23). To determine the effects of Zol on epithelial dysplasia we examined intestinal morphology at day 7, 42 and 63 of adult life following treatment with vehicle or Zol starting at either day 4 or 40 of adult life. Using $S u(H)$-lacZ; esg-Gal4, UAS-GFP reporters we labeled intestinal stem cells and enteroblasts (progenitor cells) on the basis of GFP expression driven by the escargot promoter - a marker associated with stemness. Epithelial dysplasia in the Drosophila gut is characterised by hyperproliferation, as identified by the G2/M marker phospho-Histone3 (pH3)(24) and mis-differentiation of intestinal stem cells(25). As expected, we observed an increase in intestinal stem-cell proliferation with age in vehicle treated flies as shown by the significant increase in the number of GFP+ / $\mathrm{pH} 3$ positive cells with age (Fig. $3 \mathrm{~A} \& \mathrm{~B}$ ) and an increase in the overall proportion of GFP+ cells (Fig. 3 C\&D). Both parameters are significantly decreased in female flies treated with Zol (Fig. 3 A-D).

One of the key roles played by the intestinal epithelia is to provide an impermeable barrier to the exterior environment in which damaged or dying epithelial cells are replaced with new cells generated by the ISCs. A loss of barrier function in the intestinal epithelium has been reported with age in both flies and humans. To assess intestinal integrity we performed the "Smurf assay"(26) in which blue colouring added to regular food is able to cross a compromised epithelium and stain the entire fly blue. Consistent with previous findings, the percentage of flies presenting the 'Smurf' phenotype was increased with age and is attenuated by treatment with Zol under the all-life feeding regime (Fig. 3 $\mathrm{E} \& \mathrm{~F})$.

Taken together, these data suggest that treatment with Zol throughout adult life reduces intestinal dysplasia and permeability in Drosophila. 


\section{Zol increases lifespan of flies exposed to oxidative stress through FOXO}

To elucidate the mechanism of action mediating the beneficial effects of Zol and considering the role of GTPases in longevity we hypothesized that resistance to oxidative stress was partly responsible for the increased survivorship. Therefore, flies were challenged with food containing $5 \% \mathrm{H}_{2} \mathrm{O}_{2}$, a treatment that reduces absolute lifespan and acts as a source of oxidative stress. Groups of adult, 11day old female $w^{D a h}$ flies were subjected to $5 \% \mathrm{H}_{2} \mathrm{O}_{2}$ at the same time as either Zol or Rapamycin used as a positive control(27). We also tested another group of flies pre-treated with Zol for 7 days before exposure to $5 \% \mathrm{H}_{2} \mathrm{O}_{2}$ (Fig. 4A). While the survival of flies treated with Rapamycin increased significantly compared to vehicle control, Zol-treatment did not improve survival when administered at the same time as the $5 \% \mathrm{H}_{2} \mathrm{O}_{2}$ (Fig. 4B). By contrast, survival in response to $\mathrm{H}_{2} \mathrm{O}_{2}$ was significantly increased following pre-treatment with Zol for 7 days (Fig. 4B). To determine whether the increase was due to inhibition of FPPS, flies pre-treated with Zol were also treated with the downstream metabolites of the mevalonate pathway, Farnesyl farnesol (FOH) and Geranyl geranyol (GGOH), which bypass the block in FPPS inhibition. As expected, the survival advantage provided by Zol pre-treatment was abrogated in the presence of $\mathrm{FOH}$ and GGOH demonstrating that the extension in survival is due inhibition of the mevalonate pathway (Fig. 4C). To further support the hypothesis that inhibition of FPPS is responsible for the resistance to oxidative damage, Fpps $s^{k 06103} /+$ and

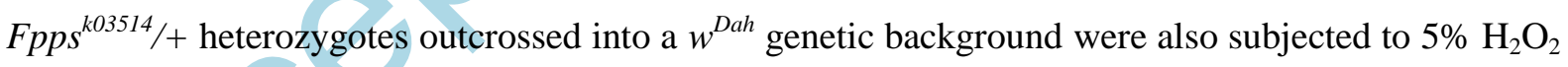
and their survival assessed. In these flies, survival was also significantly increased compared to $w^{\text {Dah }}$ controls following exposure to $\mathrm{H}_{2} \mathrm{O}_{2}$-induced oxidative stress (Fig. 4D). Taken together these data suggest that the Zol confers resistance to oxidative stress and demonstrates that these effects are mediated by inhibition of the FPPS enzyme in the mevalonate pathway.

To determine the mechanism of action downstream of the mevalonate pathway, we hypothesized that Zol may be affecting the activity of dFOXO, a factor regulating oxidative stress signals. To determine 
whether Zol protects Drosophila from oxidative damage through $\mathrm{dFOXO}$, heterozygous $d F O X O^{494} /+$ loss of function mutations(17) and $w^{\text {Dah }}$ controls were pre-treated with either Zol at the highest dose of $10 \mu \mathrm{M}$ or vehicle control-containing food for 7 days before exposure to food containing $5 \% \mathrm{H}_{2} \mathrm{O}_{2}$. While $w^{D a h}$ pre-treated with Zol showed a significant increase in lifespan, $d F O X O^{494} /+$ flies treated in the same way did not show any beneficial effect (Fig. 4E), suggesting dFOXO is required for Zol action on survival.

Given that pAKT is a known regulator of $\mathrm{FOXO}(28)$ we next determined levels of pAKT, following treatment with Zol and observed a significant decrease (Fig. 4F and eFig. 5). Furthermore, these effects were reversed by the addition of FOH and GGOH (Fig. 4 F\&G), suggesting that inhibition of pAKT is dependent on the mevalonate pathway.

Taken together these data suggest that Zol confers resistance to oxidative damage via inhibition of the mevalonate pathway through a mechanism that requires $\mathrm{dFOXO}$ for its activity.

\section{Zol enhances DNA damage repair in Drosophila upon irradiation}

DNA damage and the resulting mutations induced by ionizing radiation are at least partly caused by increased oxidative stress(29). We therefore wanted to determine whether the ability of Zol to protect against $\mathrm{H}_{2} \mathrm{O}_{2}$-induced oxidative stress would also reduce the frequency of DNA damage induced by $\mathrm{X}$-ray irradiation. In order to visualise DNA damage in an in vivo environment, we developed an assay in which endogenously expressed mRNA from the wild type white locus is knocked down by an in vivo RNAi hairpin-loop expressed within the cells of the future eye. Where knock down is successful, white mRNA is destroyed and very little pigment is produced, resulting in pale yellow eye pigmentation. Where the DNA encoding components of the GMR-Gal4,UAS-white ${ }^{R N A i}$ expression 
system are mutated, white mRNA is not destroyed and wild type levels of red pigment are produced to give a readily recognisable red eye clone (Fig. 5A) in adult Drosophila. In order to assess DNA damage levels using this reporter, larvae heterozygous for the GMR-Gal4,UAS-white ${ }^{R N A i}$ reporter were raised on food containing either carrier controls, $1 \mu \mathrm{M}$ Zol, $10 \mu \mathrm{M}$ Zol or a combination of $10 \mu \mathrm{M}$ Zol and $33 \mu \mathrm{M} \mathrm{FOH}$ and $33 \mu \mathrm{M}$ GGOH. Larvae were irradiated 96 hours after hatching using one dose of 200Gy of X-ray irradiation (Fig. 5C). Strikingly, GMR-Gal4,UAS-white RAAi $_{+}+$flies contained significantly lower frequency of red-marked mutated cells when grown on 1 or $10 \mu \mathrm{M}$ Zol-containing food compared to controls. However, when Drosophila were treated with $10 \mu \mathrm{M}$ Zol in combination with $\mathrm{FOH}$ and $\mathrm{GGOH}$, a partial reversal of this effect was observed (Fig. 5C). These data suggest that Zol is also able to protect individuals from the accumulation of mutations, via mechanism(s) that, at least in part, depend on the mevalonate pathway.

\section{DISCUSSION}

In this study we show that Zol has properties of a geroprotector, an activity mediated by its inhibition of FPPS. We show that Zol extends the lifespan and healthspan of Drosophila in absence of mineralised bone-like structures and demonstrate that it confers resistance to oxidative damage via the inhibition of FPPS in the mevalonate pathway. The median extension of lifespan by Zol is in the same range of other geroprotectors including rapamycin, with Zol extending the median lifespan of females by $14-18 \%$ when given from middle age. Rapamycin, one of the most studied geroprotectors, which positively impacts on the immune system in older patients by boosting their ability to improve flu vaccine responses(30) and also improves several healthspan parameters in mice(31), increases lifespan by $9-14 \%$ in female mice when fed late in life(32). A similar level of extension was shown in other studies testing rapamycin in Drosophila(33). 
Our results suggest that $\mathrm{Zol}$ requires $\mathrm{dFOXO}$ for its action and is associated with reduction in the levels of pAKT. These in vivo findings are in line with our previous work in human mesenchymal stem cells (hMSC) where we have shown that Zol was able to reduce the accumulation of DNA damage caused by cellular ageing or irradiation via a mechanism requiring FOXO3a, as well as reducing the accumulation of senescent markers p16 and p21 (ref(34)). In addition, Zol was able to increase the translocation of pFOXO3a to the nucleus in human mesenchymal stem cells and this was reversed by the addition of $\mathrm{FOH}$ and $\mathrm{GGOH}$, suggesting an action on the activation of FOXO3a(34). Molecules such as Rheb and Ras are small GTPases prenylated via the mevalonate pathway and upstream of TORC1 and TORC2 and therefore are likely candidates to mediate the regulation of PAKT and FOXO by Zol $(35,36)$. FOXO is a key player in ageing and has been shown to regulate several of its hallmarks including DNA damage, senescence, changes in mitochondrial function and mutation rates(37). Whilst Drosophila have a single FOXO gene (dFOXO), the human genome is more complex and encodes four different FOXO proteins, with polymorphisms in FOXO3a having been associated with exceptional longevity $(38,39)$. In addition, FOXO3a activity has been shown to reduce the effects of reactive oxygen species (ROS) production in multiple ways. For example, its expression improves the fidelity of DNA damage repair by arresting the cell cycle to allow the repair of damaged DNA(40,41). In addition, FOXO3a activation results in the repression of a large number of nuclear-encoded genes with mitochondrial function(42). As most intrinsic ROS are produced by the respiratory complexes located in the inner mitochondrial membrane, these changes in mitochondrial activity may directly influence the levels of ROS production in vivo. More work is required to understand in detail which of these mechanisms and molecular pathways Zol modulates via FOXO. In addition, it will be important to determine which of the many small GTPases modulated by the mevalonate pathway are responsible for FOXO activity. A detailed analysis of each individual tissue in a mammalian system is also required as there are important differences in response to oxidative stress not only among tissues but even within regions of the same tissues(43). 
A similar molecular mechanism mediating extension of lifespan has been described following administration of statins in C. elegans. Statins inhibits HMG-Co-A reductase in the mevalonate pathway and extends lifespan via DAF16/FOXO3a(44). However, statins are administered daily as opposed to Zol which is given once a year in patients affected by osteoporosis. This can be an advantage in terms of cost-effectiveness, especially when considering preventive interventions.

One observation we made is that the effects of Zol varied depending on time of administration, dose and sex differences that may be partly due to differences in drug uptake by males and females. In females, egg production requires higher levels of nutritional input than required by males, resulting potentially in increased food consumption and therefore increased drug uptake with consequent signs of toxicity(45). By contrast, in the FPPS mutants, where the action of the enzyme is disrupted independently of drug uptake, similar lifespan extension is observed in both sexes suggesting that the effect of mevalonate pathway inhibition on lifespan is unlikely to be sexually dimorphic. However, further work is required to understand this aspect of the work.

In addition to differences in lifespan extension, it is intriguing to note that indicators of improved health such as climbing ability and intestinal dysplasia occur even at high doses of Zol that reduce absolute lifespan (compare Fig. 1B $10 \mu \mathrm{M}$ with Fig. 3A, 63day $10 \mu \mathrm{M}$ ), demonstrating that drug treatment is able to increase healthspan independently of absolute lifespan. This observation is in line with the finding of others which shows that healthspan and lifespan are not necessarily related(46). For example, Nicotinamide has recently been shown to improve aspects of healthspan but not lifespan(47). This disconnect between healthspan and lifespan may indicate that organisms can cope with the accumulation of a certain number of defects which are sufficient to negatively affect healthspan but which do not lead directly to death. Being able to reduce these healthspan-associated deficits is of particular interest from a translational perspective as they contribute to morbidity and poor health and are responsible for significant healthcare costs. 
Another notable aspect of Zol treatment in Drosophila is the improvement in lifespan and healthspan measures following treatment that begins only in middle age (Fig. $1 \mathrm{E} \& \mathrm{~F})$. It is unclear why the effects are more prominent when the treatment starts at middle age, particularly in females. The treatment profile observed in these flies mirrors that of women affected by osteoporosis who also generally begin Zol treatment post-menopausally. In both Drosophila and humans, the effect of Zol on survival is only detectable sometime after initial treatment(48). On average, it takes 16 months before an improvement in survival is observed in postmenopausal women(48) and approximately 14 days in Drosophila (Fig. 1F). The reasons for this delayed phenotypic response are unclear but may reflect an ability by Zol to improve biological processes only when they are mildly dysregulated. However, when those mechanisms are either working at healthy levels or when their dysregulation exceeds a compensatory threshold then Zol treatment is either not needed or no longer sufficient to maintain function. Consistent with this model, Zol did not have any effect on climbing activity in geriatric flies at 70 days of age - when no activity was detected in any of the populations tested. It is possible that a similar process may also explain why a Zol-mediated extension of lifespan was not observed in Zmpste $24^{-/}$mice. In this model of Hutchinson-Guilford progeria syndrome Zol alone does not modify premature ageing while combined treatment with both Zol and statins (which inhibit the same pathway) is able to extend lifespan(49).

While evidence collected to date suggests that the effects of Zol on lifespan and healthspan in humans may be limited, it should be noted that the effects observed follow treatment with just a single yearly dose. None-the-less, retrospective analysis of patients taking Zol has not only shown a marked increase in survival but also a reduction in the frequency of death by pneumonia and cardiovascular events, suggesting broader effects unrelated to the musculoskeletal system(9). In addition, patients treated with Zol and admitted to hospital for intensive / critical care for a condition not related to osteoporosis showed reduced mortality rates relative to controls (5.2\% vs $9.1 \%$ respectively). 
Furthermore, the patients previously treated with Zol required 30\% shorter in-patient care despite being older than the control group and having higher comorbidity index(10).

Older people with frailty and multimorbidity have a reduced ability to respond to adverse events and often lose independence following major health-related incidents. Such events have significant consequences for follow up health and social care costs $(50,51)$. Indeed, there has been an increase of $18 \%$ in the number of emergency admissions of older patients between 2010/11 and 2014/15 in the UK with those patients over the age of 65 now accounting for $62 \%$ of total bed days spent in hospital. Considering average cost for a patient to stay in an NHS ward is up to $£ 400$ per day, the financial and societal benefits of improving resilience in older people and reducing length of hospital stay are huge(51).

In conclusion we have shown that inhibition of FPPS by Zol modulates mechanisms of ageing to extend lifespan and healthspan in vivo - an effect that is independent of its effects on bone. These findings are in line with the unexplained improved survival rates that have been reported recently for patients being treated with Zol - findings that highlight the substantial benefit Zol can potentially provide with only a single yearly treatment. Studies in mammalian models are now required to understand the effects of Zol on specific tissues, to define which of the FOXO-regulated mechanisms are at play and whether an infrequent administration of Zol is the best approach to elicit the strongest beneficial effects. Given that Zol is off-patent, available at low cost and displays a well understood safety profile featuring minimal side effects, we suggest that repurposing studies seeking to widen the use of Zol are likely to identify great potential for the improvement of healthspan and resilience in older people. 


\section{Acknowledgements}

We would like to thank Leanne Joes for fly stocks and valuable advice, Kath Whitley and fly room technical support staff for assistance with fly food preparation and stock maintenance. We would also like to thank the Bloomington and Vienna Drosophila stock centres for fly stocks and Hal Hebetino and Graham Russell for donating zoledronate and for helpful discussions. This work was supported by a University of Sheffield PhD studentship award.

\section{Conflicts of interest}

None

\section{Author Contributions}

IB, MZ conceived the idea, analysed the data, wrote the manuscript ZC, MZ and AA performed the experiments, analysed the data and wrote the manuscript. All authors designed the experiments, reviewed and approved the manuscript. 


\section{Data Availability}

The data that support the findings of this study are available from the corresponding author upon reasonable request. 


\section{References}

1. WHO. World report on ageing and health. Luxemburg; 2015. http://apps.who.int/iris/bitstream/handle/10665/186463/9789240694811_eng.pdf;jsessionid= 62871338D5845B327AA38A180D9919E0? sequence $=1$

2. Bellantuono I. Find drugs that delay many diseases of old age. Nature. 2018;554:293295. DOI: $10.1038 / \mathrm{d} 41586-018-01668-0$

3. Vogeli C, Shields AE, Lee TA, Gibson TB, Marder WD, Weiss KB, et al. Multiple chronic conditions: prevalence, health consequences, and implications for quality, care management, and costs. J Gen Intern Med. 2007;22 Suppl 3:391-395. DOI: 10.1007/s11606-007-0322-1

4. Maher RL, Hanlon JT, Hajjar ER. Clinical Consequences of Polypharmacy in Elderly. Expert opinion on drug safety. 2014;13:10.1517/14740338.14742013.14827660.DOI:

$10.1517 / 14740338.2013 .827660$

5. Luckman SP, Hughes DE, Coxon FP, Graham R, Russell G, Rogers MJ. Nitrogencontaining bisphosphonates inhibit the mevalonate pathway and prevent post-translational prenylation of GTP-binding proteins, including Ras. J Bone Miner Res. 1998;13:581589.DOI: $10.1359 / \mathrm{jbmr}$.1998.13.4.581

6. Weiss HM, Pfaar U, Schweitzer A, Wiegand H, Skerjanec A, Schran H. Biodistribution and plasma protein binding of zoledronic acid. Drug Metab Dispos. 2008;36:2043-2049.DOI: 10.1124/dmd.108.021071

7. Lambrinoudaki I, Vlachou S, Galapi F, Papadimitriou D, Papadias K. Once-yearly zoledronic acid in the prevention of osteoporotic bone fractures in postmenopausal women. Clin Interv Aging. 2008;3:445-451. DOI: 10.2147/cia.s2046

8. McClung M, Recker R, Miller P, Fiske D, Minkoff J, Kriegman A, et al. Intravenous zoledronic acid $5 \mathrm{mg}$ in the treatment of postmenopausal women with low bone density previously treated with alendronate. Bone. 2007;41:122-128. DOI: 10.1016/j.bone.2007.03.011

9. Colón-Emeric CS, Mesenbrink P, Lyles KW, Pieper CF, Boonen S, Delmas P, et al. Potential mediators of the mortality reduction with zoledronic acid after hip fracture. $\mathrm{J}$ Bone Miner Res. 2010;25:91-97. DOI: 10.1359/jbmr.090704

10. Lee P, Ng C, Slattery A, Nair P, Eisman JA, Center JR. Preadmission Bisphosphonate and Mortality in Critically Ill Patients. The Journal of Clinical Endocrinology \& Metabolism. 2016;101:1945-1953.DOI: 10.1210/jc.2015-3467

11. Zhang FL, Casey PJ. Protein prenylation: molecular mechanisms and functional consequences. Annu Rev Biochem. 1996;65:241-269. DOI: 10.1146/annurev.bi.65.070196.001325

12. Durán RV, Hall MN. Regulation of TOR by small GTPases. EMBO Rep. 2012;13:121-128. DOI: 10.1038/embor.2011.257

13. Moussian B, Schwarz H, Bartoszewski S, Nüsslein-Volhard C. Involvement of chitin in exoskeleton morphogenesis in Drosophila melanogaster. J Morphol. 2005;264:117-130. DOI: $10.1002 /$ jmor. 10324.

14. Kanazawa, I. Osteocalcin as a hormone regulating glucose metabolism. World journal of diabetes. 2015; 6 (18): 1345-54. DOI. 10.4239/wjd.v6.i18.1345 
15. Grandison RC, Wong R, Bass TM, Partridge L, Piper MDW. Effect of a Standardised Dietary Restriction Protocol on Multiple Laboratory Strains of Drosophila melanogaster. PLOS ONE. 2009;4:e4067.DOI: 10.1371/journal.pone.0004067

16. Spradling AC, Stern D, Beaton A, Rhem EJ, Laverty T, Mozden N, et al. The Berkeley Drosophila Genome Project gene disruption project: Single P-element insertions mutating 25\% of vital Drosophila genes. Genetics. 1999;153:135-177. PMID 10471706

17. Slack C, Giannakou ME, Foley A, Goss M, Partridge L. dFOXO-independent effects of reduced insulin-like signaling in Drosophila. Aging cell. 2011;10:735-748. DOI 10.1111/j.1474-9726.2011.00707.x

18. Choi NH, Kim JG, Yang DJ, Kim YS, Yoo MA. Age-related changes in Drosophila midgut are associated with PVF2, a PDGF/VEGF-like growth factor. Aging Cell. 2008;7:318-334. DOI: 10.1111/j.1474-9726.2008.00380.x

19. Liu H, Ma C, Moses K. Identification and functional characterization of conserved promoter elements from glass: a retinal development gene of Drosophila. Mech Dev. 1996;56:73-82. DOI: 10.1016/0925-4773(96)00512-6

20. Dietzl G, Chen D, Schnorrer F, Su K-C, Barinova Y, Fellner M, et al. A genome-wide transgenic RNAi library for conditional gene inactivation in Drosophila. Nature. 2007;448:151-156. DOI: 10.1038/nature05954

21. Shell BC, Schmitt RE, Lee KM, Johnson JC, Chung BY, Pletcher SD, et al. Measurement of solid food intake in Drosophila via consumption-excretion of a dye tracer. Sci Rep. 2018;8:11536. DOI: 10.1038/s41598-018-29813-9

22. Gargano JW, Martin I, Bhandari P, Grotewiel MS. Rapid iterative negative geotaxis (RING): a new method for assessing age-related locomotor decline in Drosophila. Exp Gerontol. 2005;40:386-395. DOI: 10.1016/j.exger.2005.02.005

23. Martins RR, McCracken AW, Simons MJP, Henriques CM, Rera M. How to Catch a Smurf? - Ageing and Beyond... In vivo Assessment of Intestinal Permeability in Multiple Model Organisms. Bio Protoc. 2018;8:e2722.DOI: 10.21769/BioProtoc.2722

24. Ren F, Wang B, Yue T, Yun E-Y, Ip YT, Jiang J. Hippo signaling regulates $<\mathrm{em}>$ Drosophila</em> intestine stem cell proliferation through multiple pathways. Proceedings of the National Academy of Sciences. 2010;107:21064-21069.

25. Biteau B, Hochmuth CE, Jasper H. JNK activity in somatic stem cells causes loss of tissue homeostasis in the aging Drosophila gut. Cell Stem Cell. 2008;3:442-455.

26. Rera M, Clark RI, Walker DW. Intestinal barrier dysfunction links metabolic and inflammatory markers of aging to death in Drosophila. Proc Natl Acad Sci U S A. 2012;109:21528-21533. DOI: 10.1073/pnas.1215849110

27. Emran S, Yang M, He X, Zandveld J, Piper MD. Target of rapamycin signalling mediates the lifespan-extending effects of dietary restriction by essential amino acid alteration. Aging (Albany NY). 2014;6:390-398. DOI: 10.18632/aging.100665

28. Das TP, Suman S, Alatassi H, Ankem MK, Damodaran C. Inhibition of AKT promotes FOXO3a-dependent apoptosis in prostate cancer. Cell Death Dis. 2016;7:e2111.DOI: 10.1038/cddis.2015.403

29. Riley PA. Free radicals in biology: oxidative stress and the effects of ionizing radiation. Int J Radiat Biol. 1994;65:27-33.DOI: 10.1080/09553009414550041

30. Mannick JB, Del Giudice G, Lattanzi M, Valiante NM, Praestgaard J, Huang B, et al. mTOR inhibition improves immune function in the elderly. Science Translational Medicine. 2014;6:268ra179-268ra179.DOI: 10.1126/scitranslmed.3009892

31. Morsli S, Bellantuono I. The use of geroprotectors to prevent multimorbidity: Opportunities and challenges. Mech Ageing Dev. 2021;193:111391.DOI: 10.1016/j.mad.2020.111391 
32. Harrison DE, Strong R, Sharp ZD, Nelson JF, Astle CM, Flurkey K, et al. Rapamycin fed late in life extends lifespan in genetically heterogeneous mice. Nature. 2009;460:392-395. DOI: $10.1038 /$ nature08221

33. Bjedov I, Toivonen JM, Kerr F, Slack C, Jacobson J, Foley A, et al. Mechanisms of life span extension by rapamycin in the fruit fly Drosophila melanogaster. Cell Metab. 2010;11:35-46. DOI: 10.1016/j.cmet.2009.11.010

34. Misra J, Mohanty ST, Madan S, Fernandes JA, Hal Ebetino F, Russell RG, et al. Zoledronate Attenuates Accumulation of DNA Damage in Mesenchymal Stem Cells and Protects Their Function. Stem Cells. 2016;34:756-767. DOI: 10.1002/stem.2255

35. Mahoney SJ, Narayan S, Molz L, Berstler LA, Kang SA, Vlasuk GP, et al. A small molecule inhibitor of Rheb selectively targets mTORC1 signaling. Nature Communications. 2018;9:548. DOI: 10.1038/s41467-018-03035-Z

36. Mendoza MC, Er EE, Blenis J. The Ras-ERK and PI3K-mTOR pathways: cross-talk and compensation. Trends Biochem Sci. 2011;36:320-328. DOI: 10.1038/s41467-018-03035$\mathrm{z}$

37. Martins R, Lithgow GJ, Link W. Long live FOXO: unraveling the role of FOXO proteins in aging and longevity. Aging Cell. 2016;15:196-207.DOI: 10.1111/acel.12427

38. Lee JC, Espéli M, Anderson CA, Linterman MA, Pocock JM, Williams NJ, et al. Human SNP links differential outcomes in inflammatory and infectious disease to a FOXO3regulated pathway. Cell. 2013;155:57-69. DOI: 10.1016/j.cell.2013.08.034

39. Donlon TA, Curb JD, He Q, Grove JS, Masaki KH, Rodriguez B, et al. FOXO3 gene variants and human aging: coding variants may not be key players. J Gerontol A Biol Sci Med Sci. 2012;67:1132-1139. DOI: 10.1093/gerona/gls067

40. Gurkar AU, Robinson AR, Cui Y, Li X, Allani SK, Webster A, et al. Dysregulation of DAF-16/FOXO3A-mediated stress responses accelerates oxidative DNA damage induced aging. Redox Biol. 2018;18:191-199.DOI: 10.1016/j.redox.2018.06.005

41. Tsai W-B, Chung YM, Takahashi Y, Xu Z, Hu MCT. Functional interaction between FOXO3a and ATM regulates DNA damage response. Nature Cell Biology. 2008;10:460-467. DOI: $10.1038 / \mathrm{ncb} 1709$

42. Ferber EC, Peck B, Delpuech O, Bell GP, East P, Schulze A. FOXO3a regulates reactive oxygen metabolism by inhibiting mitochondrial gene expression. Cell Death Differ. 2012;19:968-979. DOI: $10.1038 /$ cdd.2011.179

43. Stefanatos R, Sanz A. The role of mitochondrial ROS in the aging brain. FEBS Lett. 2018;592:743-758. DOI: 10.1002/1873-3468.12902

44. Jahn A, Scherer B, Fritz G, Honnen S. Statins Induce a DAF-16/Foxo-dependent Longevity Phenotype via JNK-1 through Mevalonate Depletion in C. elegans. Aging Dis. 2020;11:60-72.DOI: 10.14336/AD.2019.0416

45. Camus MF, Huang C-C, Reuter M, Fowler K. Dietary choices are influenced by genotype, mating status, and sex in Drosophila melanogaster. Ecology and Evolution. 2018;8:5385-5393. DOI: https://doi.org/10.1002/ece3.4055

46. Fischer KE, Hoffman JM, Sloane LB, Gelfond JA, Soto VY, Richardson AG, et al. A cross-sectional study of male and female C57BL/6Nia mice suggests lifespan and healthspan are not necessarily correlated. Aging (Albany NY). 2016;8:2370-2391.DOI: 10.18632/aging.101059

47. Mitchell SJ, Bernier M, Aon MA, Cortassa S, Kim EY, Fang EF, et al. Nicotinamide Improves Aspects of Healthspan, but Not Lifespan, in Mice. Cell Metab. 2018;27:667676.e664. DOI: 10.1016/j.cmet.2018.02.001

48. Lyles KW, Colón-Emeric CS, Magaziner JS, Adachi JD, Pieper CF, Mautalen C, et al. Zoledronic acid and clinical fractures and mortality after hip fracture. N Engl J Med. 2007;357:1799-1809.DOI: 10.1056/NEJMoa074941 
49. Varela I, Pereira S, Ugalde AP, Navarro CL, Suárez MF, Cau P, et al. Combined treatment with statins and aminobisphosphonates extends longevity in a mouse model of human premature aging. Nat Med. 2008;14:767-772. DOI: 10.1038/nm1786

50. Liotta G, Gilardi F, Orlando S, Rocco G, Proietti MG, Asta F, et al. Cost of hospital care for the older adults according to their level of frailty. A cohort study in the Lazio region, Italy. PLoS One. 2019;14:e0217829. DOI: 10.1371/journal.pone.0217829

51. National Audit Office. Discharging older patients from hospital.; 2016. https://www.nao.org.uk/wp-content/uploads/2015/12/Discharging-older-patients-fromhospital-Summary.pdf 


\section{Figure legend}

Fig. 1 Administration of Zol affects lifespan of flies

Percentage survival of (A) male and (B) female $w^{\text {Dah }}$ flies fed with food in presence or absence of Zol (1 or $10 \mu \mathrm{M})$ throughout their lives; percentage survival of (C) male and (D) female heterozygote FPPS mutant flies and $w^{\text {Dah }}$ fed with standard Drosophila food; percentage survival of (G) male and (F) female $w^{D a h}$ flies fed with food in presence or absence of Zol (1 or $\left.10 \mu \mathrm{M}\right)$ from 40 days of age. Average of 3 experiments with 100 flies/group/experiment. Log-rank (Mantel-Cox) test in Graphpad Prism used to statistically analyse the survival curves, * $\mathrm{P} \leq 0.05, * * \mathrm{P} \leq 0.01, * * * \mathrm{P} \leq 0.001, * * * * \mathrm{P}$ $\leq 0.0001$. All experiments were repeated 3 times.

Fig. 2 Flies fed with Zol shows a significant increase in climbing ability at middle age.

Lifelong treatment with Zol: (A) Percentage of male flies climbed above 10cm (high climbers) within 15 seconds. (B) Percentage of female flies climbed above $10 \mathrm{~cm}$ (high climbers) within 15 seconds. Flies treated with Zol from midlife: (C) Percentage of male flies climbed above $10 \mathrm{~cm}$ (high climbers) within 15 seconds. (D) Percentage of female flies climbed above $10 \mathrm{~cm}$ (high climbers) within 15 seconds. Data were analysed by Two-Way ANOVA and Dunnet's multiple comparison test. * $\mathrm{P} \leq$ $0.05, * * \mathrm{P} \leq 0.01, * * * \mathrm{P} \leq 0.001 * * * * \mathrm{p} \leq 0.0001$

Fig. 3 Treatment with Zol reduces epithelial dysplasia and intestinal permeability in Drosophila

(A-B) Quantification of $\mathrm{pH} 3+$ cell in the intestine of Drosophila. Flies were treated with or 1 or without $10 \mu \mathrm{M}$ of Zol from 4 days of age (A) or 40 days of age (B). At least 7 midguts were assessed per condition and per time point. (C-D) Quantification of $\% \mathrm{GFP}^{+}$cells (normalised to DAPI) in the intestine of flies treated with or without 1 or $10 \mu \mathrm{M}$ of Zol from 4 days of age (C) or 40 days of age 
(D). (E-F) Percentage of flies Smurf positive following treatment with or without 1 or $10 \mu \mathrm{M}$ of Zol starting from 4 days of age (E) or 40 days of age $(F)$ ( $>65$ per condition per timepoint). Data were analysed with one-way ANOVA and Sidak's post-hoc test (number of PH3+ cells and \%GFP ${ }^{+}$cells) and Chi square test for the analysis of the smurf assay. $* \mathrm{p}<0.05, * * \mathrm{p}<0.01, * * * \mathrm{p}<0.001$, $* * * * \mathrm{p}<0.0001$

Fig. 4 Zol increases lifespan of flies under oxidative stress through FOXO

(A) A schematic representation of the experimental design. (B) Survivorship of female $w^{\text {Dah }}$ flies fed on food containing $5 \% \mathrm{H}_{2} \mathrm{O}_{2}$. The flies were treated with $5 \% \mathrm{H}_{2} \mathrm{O}_{2}$ in combination with PBS (vehicle) or $10 \mu \mathrm{M} \mathrm{Zol} \mathrm{or} 200 \mu \mathrm{M}$ rapamycin (Rap) both given at the same time than $\mathrm{H}_{2} \mathrm{O}_{2}$. Two groups of flies were pretreated for 7-days with $10 \mu \mathrm{M}$ Zol (d4) or PBS (d4) as vehicle before addition of $\mathrm{H}_{2} \mathrm{O}_{2}(\mathrm{C})$ Survivorship of female $w^{\text {Dah }}$ flies fed with $5 \% \mathrm{H}_{2} \mathrm{O}_{2}$. The flies were pre-treated PBS+EtOH (Vehicles), $10 \mu \mathrm{M}$ Zol, or $10 \mu \mathrm{M}$ Zol in combination with $330 \mu \mathrm{M}$ FOH \& $330 \mu \mathrm{M}$ GGOH. (D) Survivorship of heterozygote female FPPS mutants: $F p p s^{k 06103} /+$ and $F p p s^{k 03514} /+$ fed with $5 \% \mathrm{H}_{2} \mathrm{O}_{2}$. $w^{\text {Dah }}$ flies either pre-treated PBS or $10 \mu \mathrm{M}$ Zol, were used as control (E) Survivorship of female $\mathrm{dFOXO}^{\Delta 94 /+}$ flies treated with $5 \% \mathrm{H}_{2} \mathrm{O}_{2} . w^{\text {Dah }}$ flies either pre-treated with $10 \mu \mathrm{M}$ Zol or PBS (vehicle) were used as control. For all survival tests, 100 flies were used in each treatment group per test, experiments were repeated 3 times with different cohorts of flies. (F) A representative example of AKT and pAKT expression in whole flies fed with Zol $(100 \mu \mathrm{M})$ for 10 days, in presence or absence of FOH $(330 \mu \mathrm{M})$, GGOH $(330 \mu \mathrm{M})$. PBS and ethanol (EtOH) were used as vehicle control. $(\mathrm{G})$ Quantification of expression level of pAKT normalised to tubulin in presence or absence of Zol $(100 \mu \mathrm{M}), \mathrm{FOH}(33 \mu \mathrm{M})$ and GGOH $(33 \mu \mathrm{M})$ for 10 days analysed by Image $(\mathrm{n}=3)$. 
Fig. 5 Zol enhances DNA damage repair in Drosophila upon irradiation

(A) A representative image of an eye from a $w^{+}$; GMR-Gal4, UAS-white RNAi fly (left) and one containing a clone of cells in the eye in which DNA damage has led to the production of red pigment (right). (B) Time line of experiment. (C) Percentage of eyes with red colonies upon 200Gy irradiation in homozygous w+; GMR-Gal4,UAS-white RNAi flies when treated with PBS (control), $1 \mu \mathrm{M}$ Zol, $10 \mu \mathrm{M}$ Zol, or $10 \mu \mathrm{M}$ Zol with $330 \mu \mathrm{M}$ FOH and GGOH. Data analysed by one way-ANOVA and Sidak post-hoc test. $* * * \mathrm{P}<0.001, * * * * \mathrm{P}<0.0001$ 
Fig.1
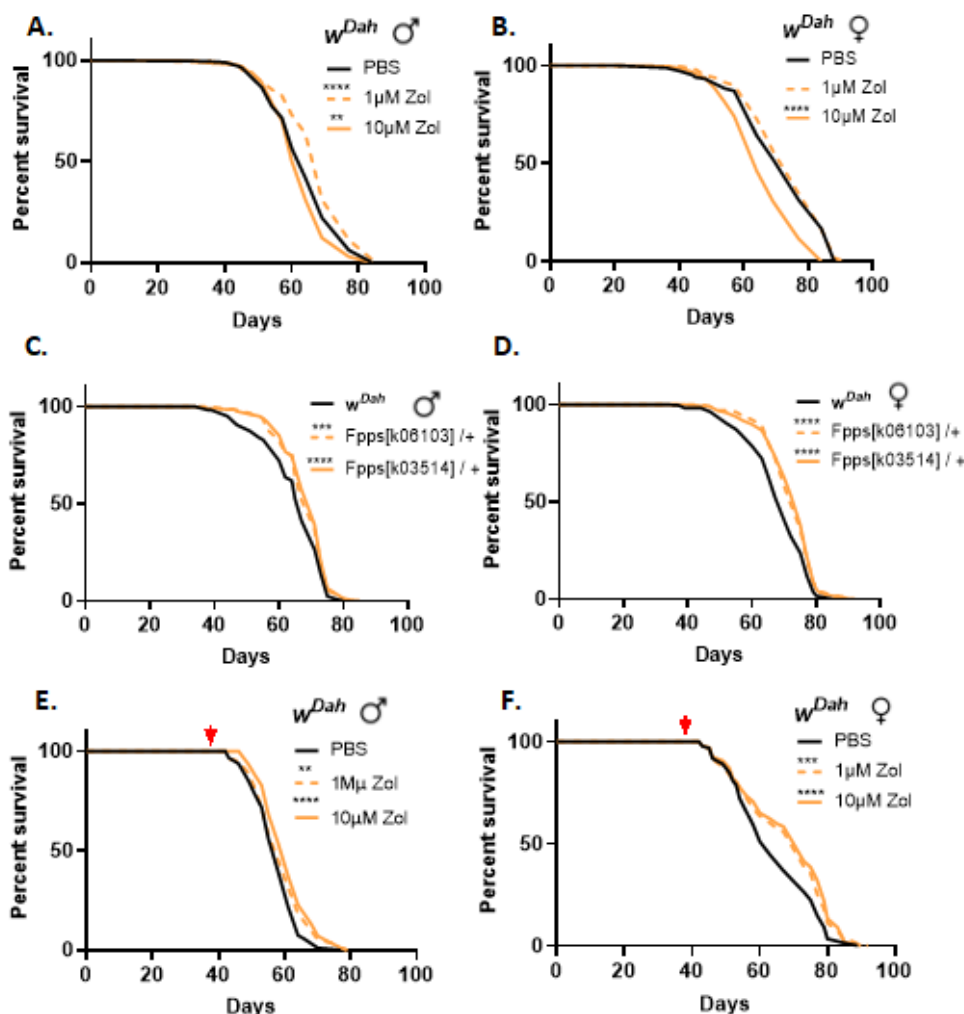
Fig. 2

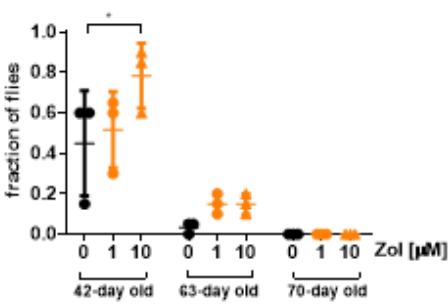

c.

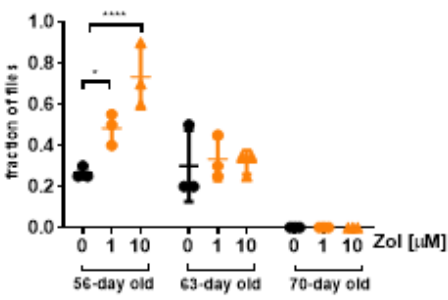

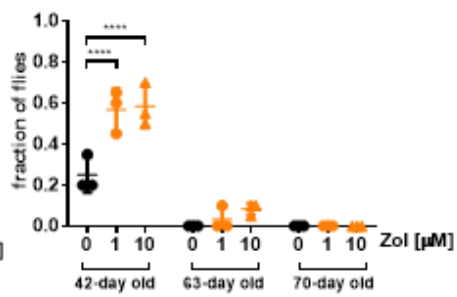

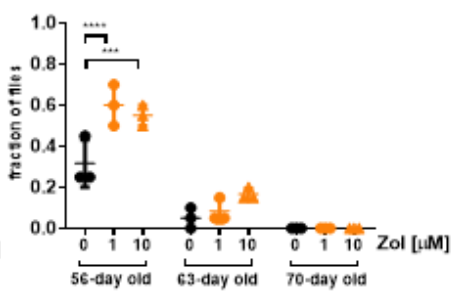

\begin{tabular}{|c|c|c|c|c|}
\hline \multicolumn{2}{|l|}{ Two-way ANOVA } & Df & Fvalue & Pvalue \\
\hline \multirow{3}{*}{$\begin{array}{l}\text { Male flies treated with } \\
\text { Zol throughout their life (Fig. } \\
\text { 2A) }\end{array}$} & Age & 2 & 57.56 & $80.0001^{*}$ \\
\hline & Treatment & 2 & 3.414 & 0.161 \\
\hline & Interaction & 4 & 1.858 & 0.0553 \\
\hline \multirow{3}{*}{$\begin{array}{l}\text { Female flies treated with } \\
\text { Zol throughout their life (Fig. } \\
\text { 2B) }\end{array}$} & Age & 2 & 162.9 & $80.0001^{*}$ \\
\hline & Treatment & 2 & 13.52 & $0.0003^{*}$ \\
\hline & Interaction & 4 & 8.238 & $0.0006^{*}$ \\
\hline \multirow{3}{*}{$\begin{array}{l}\text { Male flies treated with } \\
\text { Zol starting at middle age (Fig. } \\
\text { 2C) }\end{array}$} & Age & 2 & 68.51 & $<0.0001^{\circ}$ \\
\hline & Treatment & 2 & 7.090 & $0.0054^{*}$ \\
\hline & Interaction & 4 & 6.433 & $0.0021^{*}$ \\
\hline \multirow{3}{*}{$\begin{array}{l}\text { Female flies treated with } \\
\text { Zol starting at middle age (Fig. } \\
\text { 2D) }\end{array}$} & Age & 2 & 166.3 & $<0.0001^{*}$ \\
\hline & Treatment & 2 & 10.33 & $0.0010^{*}$ \\
\hline & Interaction & 4 & 5.833 & $0.0034^{*}$ \\
\hline
\end{tabular}


Fig.3
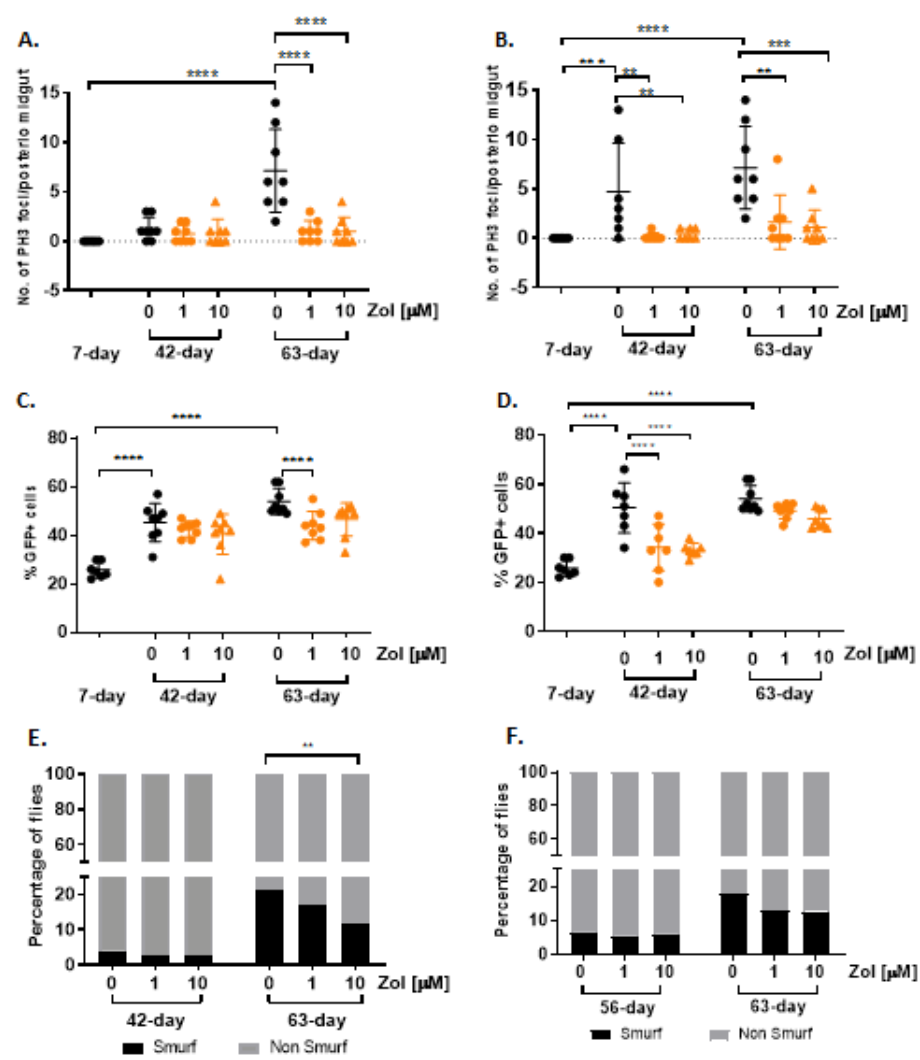
Fig.4

A.
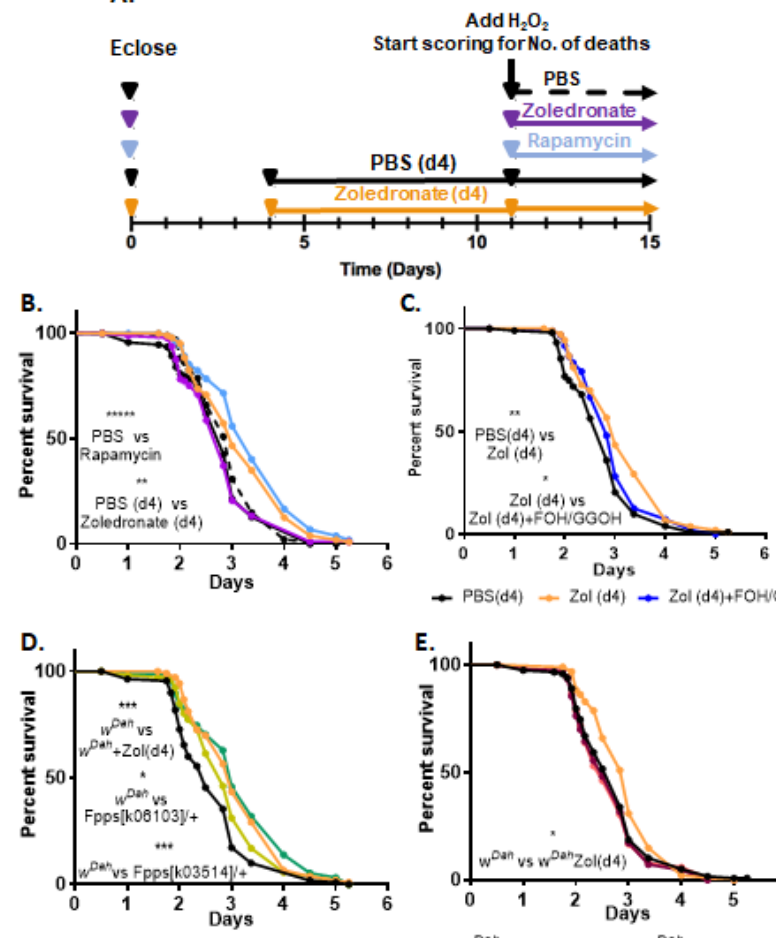

$-\mathrm{PBS}(\mathrm{d} 4) \rightarrow \mathrm{Zol}(\mathrm{d} 4)+\mathrm{Zol}(\mathrm{d} 4)+\mathrm{FOH} / \mathrm{GGOH}$

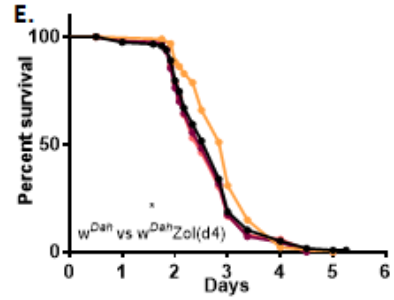

$\rightarrow w^{\circ a n}+w^{\text {aan }}+\mathrm{Zol}(\mathrm{d} 4)$
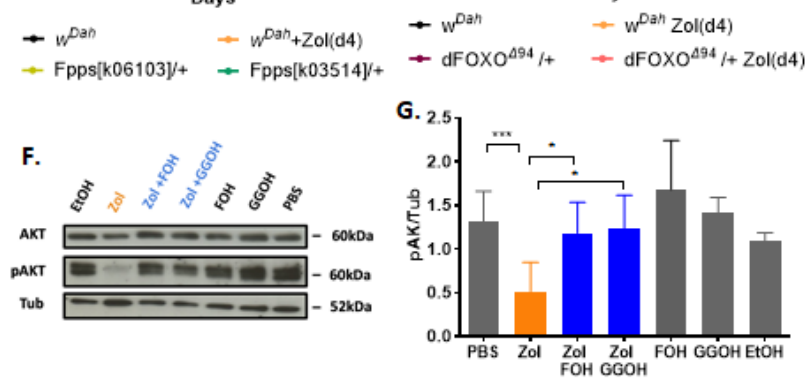
Fig. 5

A.
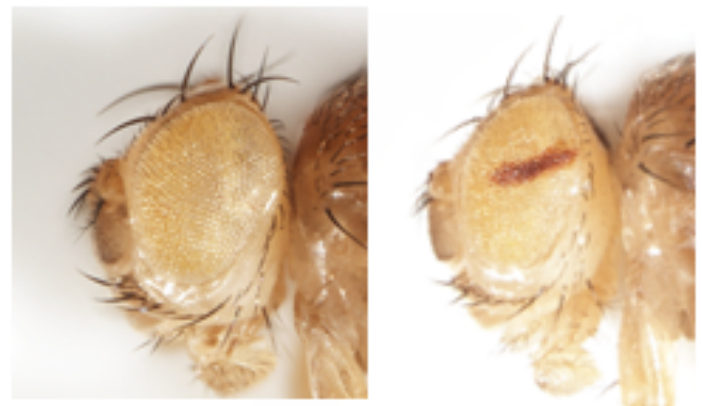

B.

$w^{*}$; GMR-Gal4 UAS-white ${ }^{\text {RNAI }}$ flies

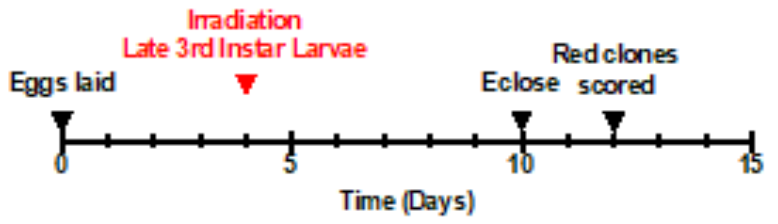

c.

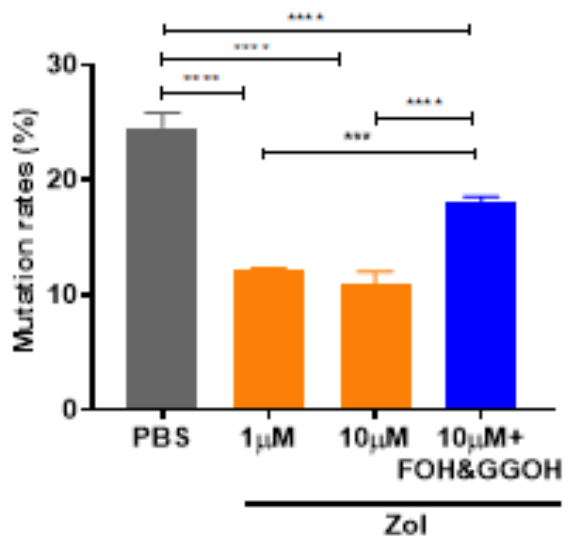

